\title{
Effects of a collaborative clinical practicum on clinical practice ability and teaching effectiveness among nursing students
}

\author{
Chun-Ja Kim¹, J ee-Won Park ${ }^{1}$, Se-Won Kang ${ }^{2}$ \\ 1. College of Nursing, Ajou University, Suwon, South Korea. 2. College of Nursing and Healthcare Science, Dong-Eui \\ University, Busan, South Korea.
}

Correspondence: Se-Won Kang. Address: College of Nursing and Healthcare Science, Dong-Eui University, 995 Eomgwangno, Jin-gu, Busan 614-714, South Korea. Email: swkang75@hotmail.com

Received: May 18, 2013

Accepted: June 6, 2013

Online Published: August 5, 2013

DOI : $10.5430 /$ jnep.v3n12p143

URL: http://dx.doi.org/10.5430/jnep.v3n12p143

\section{Abstract}

Background: This study investigates the effects of a collaborative clinical practicum for adult health nursing on clinical practice ability and teaching effectiveness among nursing students, using a non-equivalent control group pre-test-post-test design.

Methods: Participants were 52 junior nursing students taking a two-credit clinical practicum course for adult health nursing at a nursing college in South Korea. Students in the two intervention groups participated in a collaborative clinical practicum, which included a 16- or 32-h preceptorship with the usual practicum, whereas those in the control group received the usual practicum by only the instructor.

Results: The intervention group with a 32-h preceptorship had the highest score for clinical practice ability and teaching effectiveness.

Conclusions: The results justify an increase in the number of preceptorship hours for junior undergraduate students in an adult nursing practicum.

\section{Key words}

Preceptorship, Clinical practice, Teaching effectiveness

\section{I ntroduction}

Clinical practice is an essential component and a major sector of nursing education worldwide ${ }^{[1]}$. Nursing knowledge is associated with clinical practice, and academic educational achievements are attained through the nature of the applied science. One goal of nursing student clinical experience is to link theory in the classroom to real life. However, direct clinical practices have decreased for students who require a change in clinical practicum ${ }^{[2]}$.

Collaboration between academics and clinicians is an essential component of nursing education ${ }^{[3]}$. Working together in a facilitative and collaborative clinical teaching partnership enhances the support for student clinical practicum ${ }^{[4]}$. 
Collaborative clinical teaching models such as Dedicated Education Units (DEU) have been applied in clinical teaching for undergraduate students in Australia ${ }^{[3-5]}$ and the US ${ }^{[6-11]}$. However, its use has been limited in Korea.

Traditional faculty and preceptorship models have been used mostly in clinical practicum among Korean undergraduate students. The type of practicum teaching in four-year undergraduate nursing education varies slightly across Korean universities. For senior nursing students, practice teaching is performed mainly by preceptors associated with the hospital; for junior students, practice teaching is conducted mainly by instructors at the nursing college ${ }^{[8]}$. Students have less opportunity for direct clinical nursing activity when taught by instructors than preceptors. Thus, they do not receive much nursing practice knowledge, but this has changed recently ${ }^{[9]}$.

It is necessary to improve clinical practice capability and teaching effectiveness by reflecting various medical demands and environmental changes in clinical practice education in nursing. In the US, nursing students are required to complete facility-based training accredited by the Health Insurance Portability and Accountability Act, while their Korean counterparts have to complete such training accredited by the Joint Commission International and electronic medical record training. Such training helps to improve the quality of patient care and enhance patient safety.

Many studies examined nursing education components in clinical practice, including clinical practice capability of nursing students regarding practicum teaching types, such as preceptorships and clinical education partnerships ${ }^{[14,15]}$, and teaching effectiveness ${ }^{[16,17]}$. However, their participants were mostly senior nursing students, and they did not compare the educational effects of different types of teaching. Consequently, little is known about the most effective approach for teaching junior Korean nursing students. In addition, most previous studies ${ }^{[3-11]}$ on clinical practice of nursing students have been conducted in Western countries. Hence, there is insufficient information to determine the most appropriate method for improving clinical practice ability and teaching effectiveness in Korea. The present pilot study examined the effects of a collaborative clinical practicum teaching type on clinical practice ability and teaching effectiveness among Korean junior nursing students.

\section{Hypotheses}

Clinical practice ability and teaching effectiveness would be higher for junior nursing students receiving practice teaching mainly from preceptors than for those receiving practice teaching mainly from instructors.

\section{Subjects and methods}

\subsection{Study design}

A non-equivalent control group pre-test-post-test design was used. The independent variable was practice teaching type, and the dependent variables were clinical practice ability and teaching effectiveness (see Table 1). Intervention groups 1 and 2 received 16- and 32-h practice teaching, respectively, performed mainly by preceptors over a $90-\mathrm{h}$ span. The control group received traditional teaching taught by an instructor only.

Table 1. Study Design and Practicum Teaching Types

\begin{tabular}{llll}
\hline & Pre-test & Intervention & Post-test \\
\hline Control group $^{\dagger}$ & $\mathrm{O}$ & & $\mathrm{O}$ \\
Intervention group 1 $^{\ddagger}$ & $\mathrm{O}$ & $\mathrm{X} 1$ & $\mathrm{O}$ \\
${\text { Intervention group } 2^{\S}}^{\S}$ & $\mathrm{O}$ & $\mathrm{X} 2$ & $\mathrm{O}$ \\
\hline
\end{tabular}

$\dagger$ Control group, teaching by an instructor only (no preceptor), for $90 \mathrm{~h}$ (existing practice)

$\$$ Intervention group 1 , taught by a preceptor for $16 \mathrm{~h}$ and an instructor for $74 \mathrm{~h}(\mathrm{X} 1)$

$\S$ Intervention group 2 , taught by a preceptor for $32 \mathrm{~h}$ and an instructor for $58 \mathrm{~h}(\mathrm{X} 2)$ 


\subsection{Participants}

Fifty-two junior students taking a two-credit clinical practicum course for adult health nursing were recruited from a nursing college in South Korea. They were allocated to intervention groups $1(n=18)$ or $2(n=17)$ or the control group ( $n$ $=17$ ). The significance threshold was 0.05 , and effect size (f) was 0.37 in the analysis of variance (ANOVA). Power ( $1-$ $\beta$ ) was $81.4 \%$, as determined using $G *$ Power 3.1, which was sufficient. Three instructors and 10 preceptors were involved in this study. Each participant was informed of the purpose of the study and voluntarily signed an informed consent form.

\subsection{Intervention procedures}

Table 2 shows the general contents of the on-site practicum. Preceptor assignment and preceptorship focused on nursing care and skills, using nursing processes, in the intervention groups. The preceptorship included a one-on-one relationship between the preceptor and the student. Surveys on general characteristics, clinical practice ability, and teaching effectiveness were conducted before and after the practicum.

Table 2. Contents of Clinical Practice

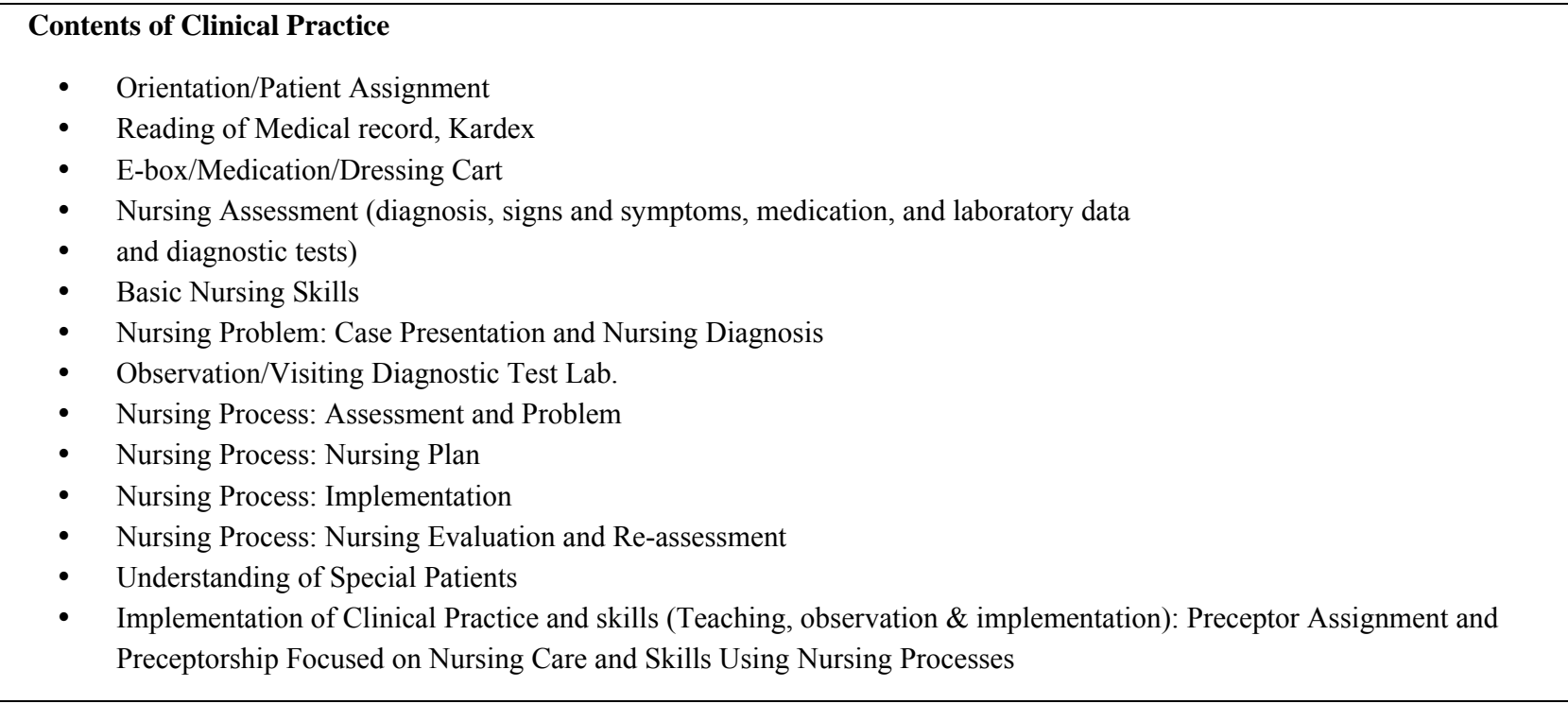

\subsection{Measurements}

Clinical practice ability. A 25-item tool with a 5-point scale developed by Lee et al. (1991) ${ }^{[18]}$ was used to assess clinical practice ability. The questions were related to direct nursing care and included measurement of vital signs, preoperative and postoperative care, formation of rapport with the patient, infection control, cooperation with relevant departments, etc. Higher scores indicated greater nursing practice capability. In this study, Cronbach's alpha was 0.94 .

Teaching effectiveness. Teaching effectiveness was measured using a 42 -item tool reconstructed by Kim et al. ${ }^{[19]}$ based on a tool developed by Reeve ${ }^{[20]}$ and Stritter, Hain, and Grimes ${ }^{[21]}$. It consisted of 5 subscales: professional knowledge and capability (5 items), interpersonal relationships and communication (13 items), usefulness as helpers (7 items), encouragement and support (5 items), and teaching methods and assessment (12 items). Higher scores indicated greater teaching effectiveness. Cronbach's alpha was 0.96 in the study by Kim et al. ${ }^{[19]}$ and 0.95 in this study.

\subsection{Data analysis}

Analyses were performed using SPSS 14.0 for Windows. General characteristics, clinical practice ability, and teaching effectiveness were analyzed with descriptive statistics (mean and SD). The homogeneity and differences in clinical practice ability and teaching effectiveness of the participants as assessed by practice teaching types were analyzed using an ANOVA. Between-group differences were analyzed using the post hoc Scheffé test. 


\section{Results}

\subsection{Homogeneity test among participants}

The average age of nursing students who participated in this study was $21.8( \pm 2.1)$ years. There were no pre-intervention between-group differences in clinical practice ability and teaching effectiveness $(F=2.908, p=.064 ; F=1.752, p=.184)$ (see Table 3). The average length of the teaching careers of the instructors and preceptors who taught nursing students was 40 and 47 months, respectively.

Table 3. Homogeneity Test Among the Three Groups

\begin{tabular}{lllllll}
\hline \multirow{2}{*}{ Variables } & \multirow{2}{*}{ Total } & $\mathbf{C G}^{\dagger}$ & $\mathbf{I G 1}^{\ddagger}$ & IG2 $^{\text {§ }}$ & \multirow{2}{*}{$\boldsymbol{F}$} & \multirow{2}{*}{$\boldsymbol{p}$} \\
\cline { 3 - 6 } & & $\mathbf{( n = 1 7 )}$ & $\mathbf{( n = 1 8 )}$ & $\mathbf{( n = 1 7 )}$ & & \\
\hline Age & $21.77(2.10)$ & $21.76(2.36)$ & $21.78(1.70)$ & $21.76(2.33)$ & .000 & 1.000 \\
Ability for clinical practice & $2.89(0.80)$ & $2.80(0.73)$ & $2.64(0.93)$ & $3.25(0.61)$ & 2.908 & .064 \\
Teaching effectiveness & $4.08(0.37)$ & $3.98(0.41)$ & $4.04(0.39)$ & $4.21(0.27)$ & 1.752 & .184 \\
\hline
\end{tabular}

$\dagger$ CG: Control group, teaching by an instructor only (no preceptor), for $90 \mathrm{~h}$ (existing practice)

\$ IG1: Intervention group 1 , taught by a preceptor for $16 \mathrm{~h}$ and an instructor for $74 \mathrm{~h}$

$\S$ IG2: Intervention group 2, taught by a preceptor for $32 \mathrm{~h}$ and an instructor for $58 \mathrm{~h}$

\subsection{Differences in clinical practice ability by practicum teaching types}

The average clinical practice ability scores of students were $2.89( \pm 0.80)$ and $2.90( \pm 0.85)$ before and after the clinical practice, respectively. The differences in the increase after the clinical practice were statistically significant among the three groups $(F=4.533, p=.016)$. In the post hoc analysis, intervention group $2(3.37 \pm 0.53)$ had a higher clinical practice ability than the control group $(2.60 \pm 0.81)$.

The following detailed items showed a statistically significant difference among groups in the analysis: measurement of vital signs $(F=4.019, p=.024)$, medication nursing $(F=3.643, p=.034)$, care of wound and pressure sores-dressing $(F=$ $3.641, p=.034)$, hot and cold therapy $(F=3.643, p=.034)$, use of a blood glucose meter $(F=4.091, p=.023)$, measurement and record of intake and output $(F=4.899, p=.011)$, postoperative care $(F=3.369, p=.043)$, follow-up care $(F=3.496, p=.038)$, nursing before and after clinical examination (diagnosis examination) $(F=3.272, p=.046)$, and lifestyle education $(F=4.147, p=.022)$ (see Table 4$)$.

\subsection{Differences in teaching effectiveness by practicum teaching types}

Students who were educated by clinical practice instructors had increased teaching effectiveness scores after clinical practice $(4.13 \pm 0.50>4.07 \pm 0.37)$, and there was a significant difference among all three groups $(F=3.416, p=.041)$. Post hoc analysis showed that intervention group 2 tended to have higher teaching effectiveness scores $(4.38 \pm 0.40)$ than intervention group $1(4.05 \pm 0.57)$ and the control group $(3.97 \pm 0.45)(p=.059$ and $p=.069$, respectively). Scores on the subscales 'interpersonal relationships and communication' $(F=3.826 ; p=.029)$ and 'encouragement and support' $(F=$ $3.178, p=.050)$ significantly differed between the three groups (see Table 5 ).

\section{Discussion}

Significant differences in clinical practice ability were observed in intervention groups. Specifically, intervention group 2, educated by a preceptor for $32 \mathrm{~h}$, showed higher clinical practice capability than the control group and intervention group 1. This implies that students' nursing practice capability is directly related to teaching time by a preceptor, which is consistent with previous studies showing that the preceptorship improved direct nursing and nursing practice capability ${ }^{[2}$, $15,22]$. 
Table 4. Differences in Clinical Practice Ability by Practicum Teaching Types

\begin{tabular}{|c|c|c|c|c|c|c|c|c|}
\hline Variables & & $\begin{array}{l}\text { Total } \\
(n=52)\end{array}$ & $\frac{C G^{a}}{(n=17)}$ & $\begin{array}{l}\text { IG1 }^{\mathrm{b}} \\
(\mathrm{n}=18)\end{array}$ & $\begin{array}{l}\text { IG2 }^{\mathrm{C}} \\
(\mathrm{n}=17)\end{array}$ & & $p$ & Post-hoc \\
\hline \multicolumn{9}{|l|}{ Subcategories } \\
\hline \multirow{2}{*}{ Vital check } & baseline & $3.35(0.71)$ & $3.24(0.83)$ & $3.22(0.73)$ & $3.59(0.51)$ & \multirow{2}{*}{4.019} & \multirow{2}{*}{.024} & $c>a$ \\
\hline & post-test & $4.25(0.71)$ & $4.24(0.75)$ & $3.94(0.73)$ & $4.59(0.51)$ & & & $(p=.024)$ \\
\hline \multirow[t]{2}{*}{ Pathophysiology } & baseline & $3.35(0.71)$ & $3.24(0.83)$ & $3.22(0.73)$ & $3.59(0.50)$ & \multirow{2}{*}{0.920} & \multirow{2}{*}{.405} & \\
\hline & post-test & $3.71(0.63)$ & $3.65(0.60)$ & $3.60(0.60)$ & $3.88(0.69)$ & & & \\
\hline \multirow{2}{*}{ Admission education } & baseline & $2.27(1.47)$ & $2.00(1.41)$ & $2.06(1.69)$ & $2.76(1.20)$ & \multirow{2}{*}{2.433} & \multirow{2}{*}{.098} & \\
\hline & post-test & $2.10(1.48)$ & $1.65(1.45)$ & $1.94(1.66)$ & $2.71(1.16)$ & & & \\
\hline \multirow{2}{*}{ Discharge education } & baseline & $2.13(1.45)$ & $1.94(1.39)$ & $1.72(1.60)$ & $2.76(1.20)$ & \multirow{2}{*}{2.826} & \multirow{2}{*}{.069} & \\
\hline & post-test & $2.12(1.51)$ & $1.59(1.41)$ & $2.00(1.71)$ & $2.76(1.20)$ & & & \\
\hline \multirow{2}{*}{ Bedside care } & baseline & $3.19(1.08)$ & $3.41(0.61)$ & $2.61(1.24)$ & $3.59(1.06)$ & \multirow{2}{*}{1.704} & \multirow{2}{*}{.192} & \\
\hline & post-test & $3.17(1.23)$ & $3.24(1.09)$ & $2.78(1.62)$ & $3.53(0.21)$ & & & \\
\hline \multirow{2}{*}{ Medication } & baseline & $2.27(1.55)$ & $2.18(1.74)$ & $1.94(1.76)$ & $2.71(0.99)$ & \multirow{2}{*}{3.643} & \multirow{2}{*}{.034} & $\mathrm{c}>\mathrm{a}$ \\
\hline & post-test & $2.38(1.47)$ & $1.88(1.58)$ & $2.17(1.65)$ & $3.12(0.78)$ & & & $(p=.045)$ \\
\hline \multirow{2}{*}{$\begin{array}{l}\text { IV preparation and } \\
\text { Care }\end{array}$} & baseline & $2.17(1.54)$ & $2.70(0.98)$ & $1.83(1.65)$ & $2.71(0.98)$ & \multirow{2}{*}{1.817} & \multirow{2}{*}{.173} & \\
\hline & post-test & $2.23(1.49)$ & $1.71(1.57)$ & $2.33(1.57)$ & $2.65(1.22)$ & & & \\
\hline Dreccing & baseline & $1.87(1.57)$ & $1.35(1.50)$ & $1.94(1.80)$ & $2.29(1.31)$ & 3641 & 034 & $c>a$ \\
\hline Dressing & post-test & $1.83(1.52)$ & $1.06(1.30)$ & $2.11(1.68)$ & $2.29(1.31)$ & 3.041 & .034 & $(p=.054)$ \\
\hline Hot and cold therany & baseline & $3.02(1.32)$ & $3.06(1.30)$ & $2.78(1.63)$ & $3.24(0.97)$ & 3043 & 026 & $c>a$ \\
\hline Hot and cold therapy & post-test & $2.98(1.42)$ & $2.35(1.66)$ & $2.94(1.47)$ & $3.65(0.70)$ & 0.943 & .020 & $(p=.026)$ \\
\hline Nursing record & baseline & $3.21(0.84)$ & $3.40(0.87)$ & $2.94(0.94)$ & $3.41(0.87)$ & 0658 & 523 & \\
\hline & post-test & $3.00(1.17)$ & $3.00(1.22)$ & $2.78(1.16)$ & $3.24(1.14)$ & & & \\
\hline F-hox medication & baseline & $2.63(0.92)$ & $2.82(1.01)$ & $2.39(0.98)$ & $2.82(1.01)$ & 2125 & 130 & \\
\hline E-box meacation & post-test & $2.83(1.02)$ & $2.59(1.27)$ & $2.67(0.90)$ & $3.24(0.75)$ & 2.125 & .130 & \\
\hline Using olucometer & baseline & $4.19(0.77)$ & $4.29(0.59)$ & $3.83(0.99)$ & $4.47(0.51)$ & 4001 & 023 & $c>b$ \\
\hline Using a giucometer & post-test & $4.21(0.89)$ & $4.18(0.64)$ & $3.15(1.15)$ & $4.65(0.61)$ & 4.091 & .023 & $(p=.023)$ \\
\hline Intake \& Outnut & baseline & $2.87(1.43)$ & $2.82(1.24)$ & $2.39(1.75)$ & $3.41(1.06)$ & 4899 & 011 & $c>a$ \\
\hline 1Itake \& Vutput & post-test & $2.88(1.53)$ & $2.12(1.62)$ & $2.89(1.45)$ & $3.65(1.17)$ & & & $(p=.011)$ \\
\hline Pre_on care & baseline & $2.02(1.48)$ & $2.65(1.27)$ & $1.72(1.60)$ & $2.65(1.22)$ & 2025 & 063 & \\
\hline Pre-op care & post-test & $2.08(1.55)$ & $1.53(1.54)$ & $2.17(1.82)$ & $2.65(1.16)$ & 2.925 & .003 & \\
\hline Poct on core & baseline & $1.98(1.43)$ & $1.65(1.37)$ & $1.72(1.60)$ & $2.59(1.18)$ & 3260 & 043 & $c>a$ \\
\hline Post-op care & post-test & $2.12(1.59)$ & $1.41(1.42)$ & $2.17(1.82)$ & $2.76(1.25)$ & 3.009 & .043 & $(p=.043)$ \\
\hline Prenaration care & baseline & $2.13(1.50)$ & $2.65(1.27)$ & $1.72(1.60)$ & $2.65(1.22)$ & 2452 & 097 & \\
\hline Preparanon care & post-test & $2.12(1.59)$ & $1.53(1.54)$ & $2.11(1.77)$ & $2.65(1.16)$ & 2.452 & .091 & \\
\hline Follow un care & baseline & $2.08(1.51)$ & $1.76(1.56)$ & $1.89(1.68)$ & $2.59(1.18)$ & 3.406 & 038 & $c>a$ \\
\hline голо口w-up cale & post-test & $2.17(1.57)$ & $1.53(1.55)$ & $2.11(1.78)$ & $2.88(1.05)$ & 0.490 & .000 & $(p=.039)$ \\
\hline Phycical exam & baseline & $2.75(0.92)$ & $2.88(0.69)$ & $2.61(1.24)$ & $2.88(0.69)$ & 114 & 327 & \\
\hline Pnysical exam & post-test & $2.73(1.30)$ & $2.59(1.46)$ & $2.50(1.54)$ & $3.12(0.69)$ & 1.144 & .321 & \\
\hline Ruilding rannort & baseline & $3.77(0.75)$ & $4.06(0.65)$ & $3.78(0.73)$ & $4.06(0.65)$ & 1725 & 180 & \\
\hline Builaing rapport & post-test & $3.88(0.94)$ & $4.06(0.75)$ & $3.56(1.14)$ & $4.06(0.82)$ & $1 . / 25$ & .189 & \\
\hline Kardey/chart reodino & baseline & $4.04(0.71)$ & $4.18(0.72)$ & $4.00(0.76)$ & $4.18(0.72)$ & 821 & 173 & \\
\hline Nardex/chant reading & post-test & $4.06(0.72)$ & $4.29(0.58)$ & $3.83(0.78)$ & $4.06(0.74)$ & 1.021 & .173 & \\
\hline Dironocic eyam & baseline & $2.48(1.43)$ & $2.35(1.41)$ & $2.44(1.72)$ & $2.65(1.17)$ & 27,20 & 046 & $c>a$ \\
\hline Diagnosis exam & post-test & $2.06(1.61)$ & $1.41(1.62)$ & $2.00(1.68)$ & $2.76(1.30)$ & 3.272 & .046 & $(p=.047)$ \\
\hline Infection car & baseline & $3.94(0.89)$ & $4.12(0.78)$ & $3.78(1.16)$ & $4.12(0.78)$ & 0250 & 773 & \\
\hline Hine tivil care & post-test & $3.88(1.23)$ & $3.76(1.52)$ & $3.83(1.15)$ & $4.06(1.02)$ & 0.253 & .173 & \\
\hline
\end{tabular}


Table 4. (continued.)

\begin{tabular}{|c|c|c|c|c|c|c|c|c|}
\hline Variables & & $\begin{array}{l}\text { Total } \\
(n=52)\end{array}$ & $\frac{\mathrm{CG}^{\mathrm{a}}}{(\mathrm{n}=17)}$ & $\begin{array}{l}\text { IG1 }^{b} \\
(n=18)\end{array}$ & $\begin{array}{l}\text { IG2 }^{\mathrm{C}} \\
(\mathrm{n}=17)\end{array}$ & $-F$ & $p$ & Post-hoc \\
\hline \multirow{2}{*}{ Lifestyle education } & baseline & $3.44(1.06)$ & $3.65(0.70)$ & $3.06(1.55)$ & $3.65(0.49)$ & \multirow{2}{*}{4.147} & \multirow{2}{*}{.022} & $c>b$ \\
\hline & post-test & $3.88(1.23)$ & $3.76(1.52)$ & $3.83(1.15)$ & $4.06(1.03)$ & & & $(p=.031)$ \\
\hline \multirow{2}{*}{$\begin{array}{l}\text { Communication with } \\
\text { patient's family }\end{array}$} & baseline & $3.70(0.95)$ & $3.82(0.88)$ & $3.67(1.08)$ & $3.82(0.88)$ & \multirow{2}{*}{1.829} & \multirow{2}{*}{.171} & \\
\hline & post-test & $3.69(0.96)$ & $3.71(0.92)$ & $3.39(1.09)$ & $4.00(0.79)$ & & & \\
\hline \multirow{2}{*}{$\begin{array}{l}\text { Cooperation with } \\
\text { other department }\end{array}$} & baseline & $2.60(1.56)$ & $3.35(0.86)$ & $1.89(1.77)$ & $3.35(0.86)$ & \multirow{2}{*}{2.669} & \multirow{2}{*}{.079} & \\
\hline & post-test & $2.77(1.46)$ & $2.94(1.14)$ & $2.17(1.68)$ & $3.24(1.34)$ & & & \\
\hline \multirow{2}{*}{ Total } & baseline & $2.89(0.80)$ & $2.80(0.73)$ & $2.64(0.93)$ & $3.25(0.61)$ & \multirow{2}{*}{4.533} & \multirow{2}{*}{.016} & $\begin{array}{l}c>a \\
(p=.024)\end{array}$ \\
\hline & post-test & $2.90(0.85)$ & $2.60(0.81)$ & $2.75(0.97)$ & $3.37(0.53)$ & & & $\begin{array}{l}c>b \\
(p=.078)\end{array}$ \\
\hline
\end{tabular}

Table 5. Differences in Teaching Effectiveness by Practicum Teaching Types

\begin{tabular}{|c|c|c|c|c|c|c|c|c|}
\hline \multirow{2}{*}{ Variables } & & \multirow{2}{*}{$\begin{array}{l}\text { Total } \\
(n=52)\end{array}$} & \multirow{2}{*}{$\begin{array}{l}\mathrm{CG}^{\mathrm{a}} \\
(\mathrm{n}=17) \\
\end{array}$} & \multirow{2}{*}{$\begin{array}{l}\text { IG1 }^{\mathrm{b}} \\
(\mathrm{n}=18) \\
\end{array}$} & \multirow{2}{*}{$\begin{array}{l}\text { IG }^{\mathrm{C}} \\
(\mathrm{n}=17)\end{array}$} & \multirow{2}{*}{$\boldsymbol{F}$} & \multirow{2}{*}{$p$} & \multirow{2}{*}{ Post-hoc } \\
\hline & & & & & & & & \\
\hline \multicolumn{9}{|l|}{ Subfactors } \\
\hline \multirow{2}{*}{$\begin{array}{l}\text { Professional knowledge } \\
\text { and competence }\end{array}$} & baseline & $4.13(0.41)$ & $4.05(0.42)$ & $4.18(0.42)$ & $4.15(0.40)$ & \multirow{2}{*}{2.328} & \multirow{2}{*}{.108} & \\
\hline & post-test & $4.17(0.48)$ & $4.05(0.44)$ & $4.09(0.53)$ & $4.36(0.42)$ & & & \\
\hline \multirow{2}{*}{$\begin{array}{l}\text { Interpersonal } \\
\text { relationships } \\
\text { and communication }\end{array}$} & baseline & $3.97(0.40)$ & $3.88(0.46)$ & $3.97(0.43)$ & $4.07(0.28)$ & \multirow{2}{*}{3.826} & \multirow{2}{*}{.029} & $c>a$ \\
\hline & post-test & $4.13(0.57)$ & $3.92(0.47)$ & $4.05(0.57)$ & $4.41(0.56)$ & & & $(p=.036)$ \\
\hline \multirow{2}{*}{$\begin{array}{l}\text { Availability as a } \\
\text { supporter }\end{array}$} & baseline & $4.13(0.42)$ & $4.07(0.46)$ & $4.12(0.47)$ & $4.21(0.31)$ & \multirow{2}{*}{1.880} & \multirow{2}{*}{.163} & \\
\hline & post-test & $4.42(0.36)$ & $4.10(0.62)$ & $4.09(0.67)$ & $4.42(0.36)$ & & & \\
\hline \multirow{2}{*}{ Encourage and support } & baseline & $4.02(0.51)$ & $3.86(0.52)$ & $3.92(0.48)$ & $4.27(0.45)$ & \multirow{2}{*}{3.178} & \multirow{2}{*}{.050} & $c>b$ \\
\hline & post-test & $4.07(0.56)$ & $3.95(0.48)$ & $3.93(0.58)$ & $4.34(0.54)$ & & & $(p=.089)$ \\
\hline \multirow{2}{*}{$\begin{array}{l}\text { Teaching method } \\
\text { and evaluation }\end{array}$} & baseline & $4.16(0.36)$ & $4.06(0.35)$ & $4.08(0.39)$ & $4.33(0.30)$ & \multirow{2}{*}{2.807} & \multirow{2}{*}{.070} & $c>a$ \\
\hline & post-test & $4.12(0.52)$ & $3.95(0.43)$ & $4.06(0.62)$ & $4.35(0.43)$ & & & $(p=.083)$ \\
\hline \multirow{3}{*}{ Total } & & & & & & \multirow{3}{*}{3.416} & \multirow{3}{*}{.041} & $c>a$ \\
\hline & baseline & $4.08(0.37)$ & $3.98(0.41)$ & $4.04(0.39)$ & $4.21(0.27)$ & & & $(p=.059)$ \\
\hline & post-test & $4.13(0.50)$ & $3.97(0.45)$ & $4.05(0.57)$ & $4.38(0.40)$ & & & $\begin{array}{l}\mathrm{c}>\mathrm{b} \\
(p=.069)\end{array}$ \\
\hline
\end{tabular}

Measurement of vital signs, medication, care of wound and pressure sores-dressing, hot and cold therapy, use of a blood glucose meter, measurement and record of intake and output, postoperative care, follow-up care, nursing before and after clinical examination, and lifestyle education significantly differed across the three types of practicum teaching. Because there were limitations with respect to the direct treatment of a patient or nursing activities with an instructor associated with the nursing college, these issues were complemented by a preceptor, which resulted in the differences noted. Therefore, it is believed that even partial utilization of a preceptor could improve clinical practice capability.

Differences in teaching effectiveness across the types of practicum teaching were also observed. Intervention group 2 (32 $\mathrm{h}$ of teaching by a preceptor) tended to score higher than intervention group 1 (16 h of teaching by a preceptor) and the control group (no teaching by a preceptor). This finding accords with the results of previous studies that examined the teaching effectiveness of preceptorship ${ }^{[16,23]}$. The areas in which an instructor was not adequate were complemented by a preceptor; thus, it is hypothesized that under these conditions, nursing students experienced a higher teaching effectiveness. The largest difference among the groups was in the subscale 'interpersonal relationships and communi- 
cation'; consensus and trust were formed between the practice teachers and students through one-on-one teaching by the preceptor, and a desirable communication capability was directly observed.

The intervention groups showed higher clinical practice capability and teaching effectiveness, which implies that partial utilization of preceptorship positively affects practice improvement. The intervention groups displayed higher clinical practice capability and teaching effectiveness than the control group. This effect increased with the duration of teaching by a preceptor. Therefore, we propose that gradual introduction of preceptorships to the curriculum for junior nursing students at a four-year nursing college can help students achieve on-site practice educational goals. In addition, policy to enhance clinical practice teaching through association with a nursing college and a hospital should be established.

\section{Nursing implications}

The findings indicate that a collaborative clinical practicum including preceptorship can help to increase clinical practice ability and teaching effectiveness among junior nursing students. Further, the number of preceptorship hours for junior undergraduate students in an adult nursing practicum should be increased. Longitudinal studies with larger samples should be conducted in the future to generalize these findings and to better understand the effect of such a practicum in other settings.

\section{References}

[1] Wotton K, Gonda J. Clinician and student evaluation of a collaborative clinical teaching model. Nurse Education in Practice. 2004; 4: 120-127. http://dx.doi.org/10.1016/S1471-5953(03)00033-7

[2] Kang YS, Cho H, Roh YS, Boo EH, Ahn KH. The effects of cooperative clinical nursing education program on the competency and satisfaction of nursing students in clinical practice. Journal of Korean Academy Society of Nursing Education. 2006; 12(1): 13-20.

[3] Gonda J, Wotton K, Edgecombe K, Mason P. Dedicated education units: 2. An evaluation. Contemporary Nurse. 1999 ; 8: $172-176$. PMid:11141790 http://dx.doi.org/10.5172/conu.1999.8.4.172

[4] Henderson A, Heel A, Twentyman M, Lloyd B. Pre-test and post-test evaluation of students' perceptions of a collaborative clinical education model on the learning environment. The Australian Journal of Advanced Nursing: A Quarterly Publication of the Royal Australian Nursing Federation. 2006; 23: 8-13.

[5] Grealish L, Bail K, Ranse K. 'Investing in the future': residential aged care staff experiences of working with nursing students in a 'community of practice'. Journal of Clinical Nursing. 2010; 19: 2291-2299. PMid:20529165

[6] Ranse K, Grealish L. Nursing students' perceptions of learning in the clinical setting of the dedicated education unit. Journal of Advanced Nursing. 2007; 58: 171-179. PMid:17445020 http://dx.doi.org/10.1111/j.1365-2648.2007.04220.x

[7] Murray T, Macintyre R, Teel, C. An analysis of partnership performance: the St. Johns Mercy Medical Center-Saint Louis University School of Nursing dedicated education unit project. Journal of Professional Nursing: Official Journal of the American Association of Colleges of Nursing. 2011; 27: e58-e63. PMid:22142928 http://dx.doi.org/10.1016/j.profnurs.2011.08.005

[8] McKown T, McKown L, Webb S. Using quality and safety education for nurses to guide clinical teaching on a new dedicated education unit. Journal of Nursing Education. 2011; 50: 706-710. PMid:22007711 http://dx.doi.org/10.3928/01484834-20111017-03

[9] Miller T. The dedicated education unit: a practice and education partnership. Nursing Leadership Forum. 2005 ; 9: 169-173. PMid:16259103

[10] Moscato S, Miller J, Logsdon K, Weinberg S, Chorpenning L. Dedicated education unit: an innovative clinical partner education model. Nursing Outlook. 2007; 55: 31-37. PMid:17289465 http://dx.doi.org/10.1016/j.outlook.2006.11.001

[11] Mulready-Shick J, Kafel K, Banister G, Mylott L. Enhancing quality and safety competency development at the unit level: an initial evaluation of student learning and clinical teaching on dedicated education units. Journal of Nursing Education. 2009; 48: 716-719. PMid:20000256 http://dx.doi.org/10.3928/01484834-20091113-11

[12] Korean Accreditation Board of Nursing. 2010 Symposium for Nursing Education Accreditation: Second-session. Korean Accreditation Board of Nursing. 2010.

[13] Happell B. A model of preceptorship in nursing: reflecting the complex functions of the role. Nursing Education Perspectives. 2009; 30: 372-376. PMid:19999939

[14] Ryan C, Shabo B, Tatum K. Using experienced clinicians to facilitate clinical education. Nurse Educator. 2011; 36: 165-170. PMid:21670636 http://dx.doi.org/10.1097/NNE.0b013e31821fdbb2

Published by Sciedu Press 
[15] Ohrling K, Hallberg I. Student nurses' lived experience of preceptorship: Part 2: the preceptor-preceptee relationship. International Journal of Nursing Studies. 2000; 37: 25-36. http://dx.doi.org/10.1016/S0020-7489(99)00055-3

[16] Choi KO, Jo HS, Kim CY. The teaching effectiveness of preceptorship on clinical nursing education. Journal of Gachongil College. 2001; 29: 51-58.

[17] Jowett R, McMullan M. Learning in practice-practice educator role. Nurse Education in Practice. 2007; 7: 266-271. PMid:17689452 http://dx.doi.org/10.1016/j.nepr.2006.08.008

[18] Lee WH, Kim CJ, Yoo JS, Huh HK, Kim KS, Lim SM. A study of development of instrument about ability of clinical practice for nursing students. Korean Journal of Nursing Science. 1991; 13: 17-29.

[19] Kim MA, Kim HS, Choi SS, Kim YY, Ahn YH, Han MJ. et al. A study of preceptor's teaching effectiveness on new entry nurse orientation program. Clinical Nursing Research. 1998; 3: 5-33.

[20] Reeve MM. Development of an instrument to measure effectiveness of clinical instructors. Journal of Nursing Education. 1994; 33(1): 15-20. PMid:8133330

[21] Stritter FT, Hain JD, Grimes DA. Clinical teaching reexamined. Journal of Medical Education. 1973; 50: 876-882.

[22] Kim KH. Clinical competence among senior nursing students after their preceptorship experiences. Journal of Professional Nursing. 2007; 23: 369-375. PMid:18053963 http://dx.doi.org/10.1016/j.profnurs.2007.01.019

[23] Choi KO, Jo HS, Kim CY, Kim BY, Chang SJ. The teaching effectiveness of preceptorship and satisfaction of student for clinical practice on nursing education. Journal of Korean Academy of Nursing Administration. 2002; 8(1): 73-83. 